\title{
Primary Aldosteronism Due to a Sub Centimeter Unilateral Adrenal Adenoma
}

\section{Manov A*, Kaur A and Hatharasinghe A}

Associate Program Director of Internal Medicine, University of Nevada, USA

*Corresponding author: Andrey Manov, Associate Program Director of Internal Medicine, Sunrise health Consortium GME Program, Las Vegas, NV and Professor, University of Nevada, Reno Medical School, NV. 5292 Painted Sunrise Dr., Las Vegas, NV, 89149, Tel: 817-676-7605; Email: andrepenev@gmail.com

\section{Case Report}

Volume 4 Issue 2

Received Date: November 10, 2020

Published Date: November 25, 2020

DOI: $10.23880 /$ oaje-16000154

\section{Abstract}

We have described a 44-year old man with past medical history of resistant hypertension on 4-antihypertensive medications including diuretic. He has not been investigated for secondary causes of hypertension despite having elevated blood pressure for 10-years and low normal Potassium level. We started the work up for secondary causes of his HTN and proved by assessing the aldosterone. To plasma renin activity ratio of more than 20 with elevate aldosterone in the blood, sodium load suppression test the existence of primary hyperaldosteronism. The cause as per the CT and MRI of the abdomen and following adrenalvein sampling was found to be right adrenal gland hypersecreting adenoma. We referred the patient for surgery and started treating the patient with mineralocorticoid antagonist with improvement of the blood pressure. Current recommendation about screening and diagnosing primary hyperaldosteronism were discussed as well as the deleterious effect of HTN due to hyperaldosteronism.

Keywords: Plasma Aldosterone; Plasma Renin Activity; Primary Hyperaldosteronism; Adrenal Vein Sampling

Abbreviations: AACE: American Association of the Clinical Endocrinologists; MRA: Mineralocorticoid Receptor Antagonists; APAs: Aldosterone Producing Adenomas.

\section{Introduction}

Primary hyperaldosteronism is one of the underdiagnosed causes of hypertension. The studies showed an incidence around $11 \%$ of patients with newly diagnosed HTN. The sporadic causes of primary hyperaldosteronism are the most common cause of the disease-bilateral hyperplasia of both adrenal glands is the cause in $60-70 \%$ of the sporadic cases of the disease and the unilateral aldosterone producing adenomas is accounting for $30-40 \%$ of the sporadic causes of the disease. Much less common causes of sporadic primary hyperaldosteronism are the adrenal carcinomas producing aldosterone or unilateral adrenal hyperplasia. There are familial causes of primary hyperaldosteronism as well which are very rare.

The Endocrine Society recommends primary aldosterone screening for people who meet one of the following criteria [1]:

- Those who have sustained blood pressure above $150 / 100$ in three separate measurements taken on different days.

- People who have hypertension resistant to three conventional antihypertensive drugs.

- People whose hypertension is controlled with four or more medications.

- People with hypertension and low levels of potassium in the blood. 
- Those who have hypertension and a mass on the adrenal gland called an adrenal incidentaloma.

- People with both hypertension and sleep apnea.

- People with hypertension and a family history of early-onset hypertension or stroke before age 40.

- All hypertensive first-degree relatives of patients with primary aldosteronism.

The consequences of hyperaldosteronism come from the effects it has on systemic blood pressure, blood vessels, inflammation, oxidative stress, glucose intolerance due to hypokalemia with resulting dyslipidemia, thrombosis, fibrosis resulting in atherosclerosis, heart failure, stroke, transient ischemic attack, arrhythmia, chronic renal disease, heart failure and myocardial infarction (Figure 1). Patients with primary aldosteronism are at much higher risk than other patients with essential hypertension for heart and kidney damage and all of the above mentioned complications [2-6]. Serious cardiovascular effects, having "higher rates of card patients with primary hypertension having the same degree of high blood pressure" [3]. Other risks of hyperaldosteronism include stroke, MI and atrial fibrillation [5].

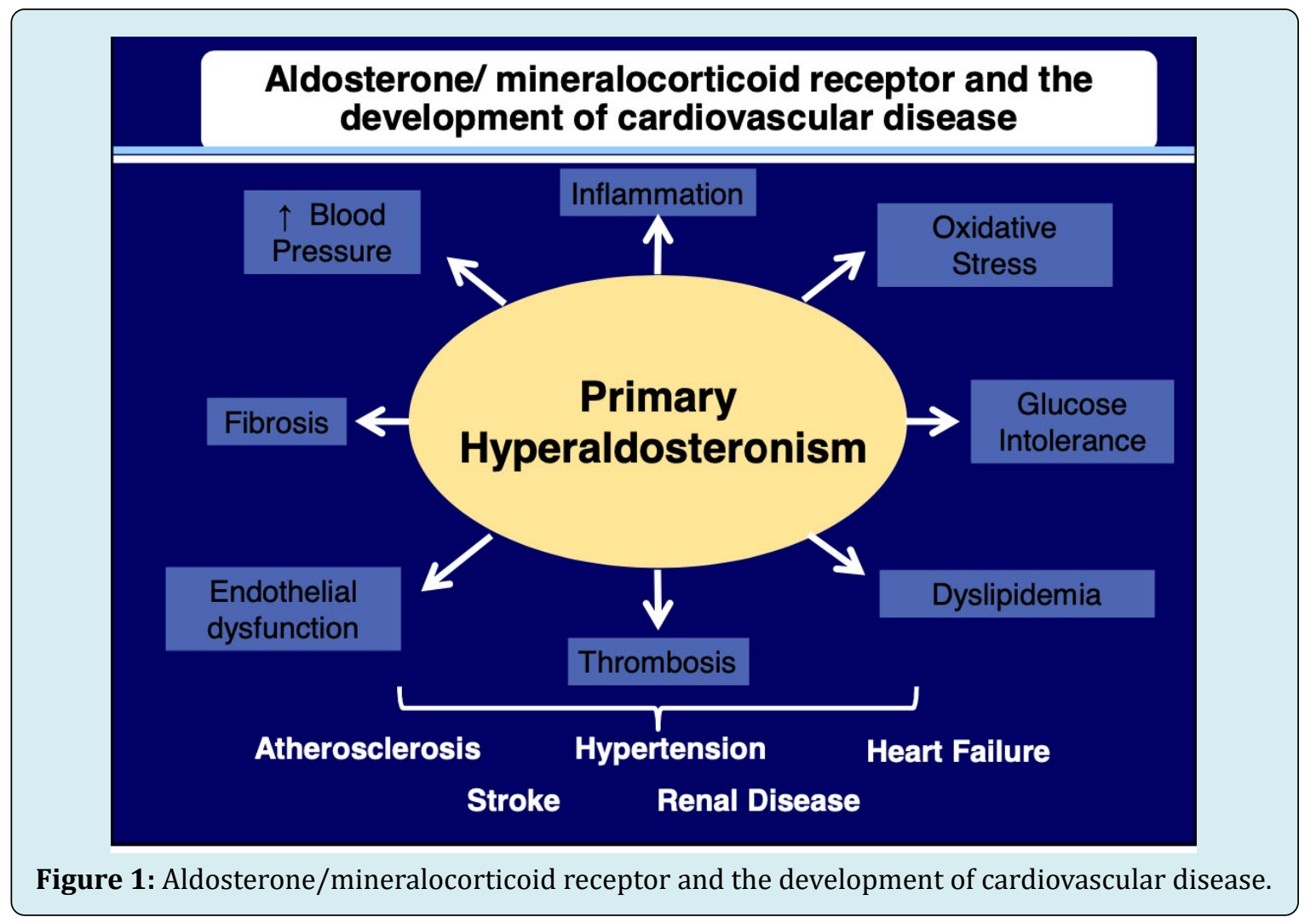

On the above figure 1 are described the mechanisms of the deleterious effects of hyperaldosteronism and why having HTN due to primary hyperaldosteronism is much more dangerous for the patient than having just essential HTN. Given into consideration that this happens in 1 in 10 patients diagnosed with essential HTN we wanted to emphasize the need for detailed screening test for the disease as required by American Association of the clinical endocrinologist's guidelines (AACE). Following the recommended guidelines by AACE by primary care physicians is of critical importance to early diagnose HTN due to hyper aldosteronism and avoid higher rate of cardiovascular and renal complications compare to such due to essential HTN.
The effects of hypertension due to excess of aldosterone were looked into in a study that investigated changes in left ventricular anatomy in patients with essential hypertension versus those having high blood pressure due to primary aldosteronism. In patients with primary aldosteronism, aldosterone causing the suppression of the renin-angiotensin system is associated with both increased left ventricular mass and significant changes of left ventricular diastolic filling pressures. The former changes appear to be reversible on removal of the cause of excessive aldosterone production. After 1 year of follow-up, highly significant decreases of left ventricular wall thickness and mass were observed in patients treated with surgical excision of an aldosterone- 


\section{Open Access Journal of Endocrinology}

producing tumor, but not in those with medical therapy.

On Figure 2 is described the suggested by AACE guidelines work up to prove primary hyperaldosteronism in patients with Hypertension. We need to keep in mind that sometimes the adrenal vein sampling is not successful in up to $25 \%$ of the patients due to inability to be cannulated right adrenal vein.

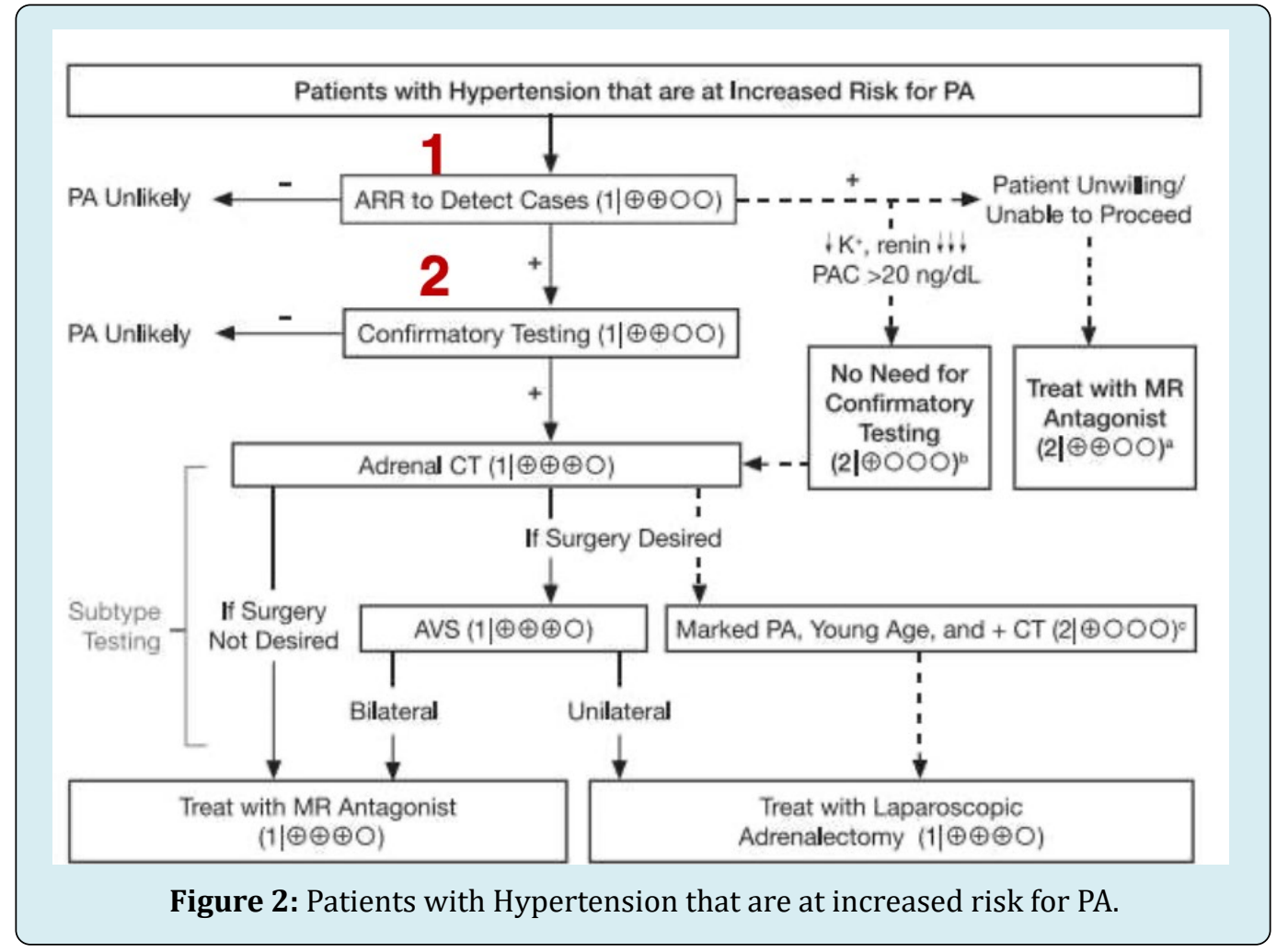

\section{Case Report}

Our patient was 44-year-old white male with hypertension (diagnosed at age 34), obesity, mixed connective tissue disease treated with methotrexate, and history of a childhood benign brain tumor (resected as a child) that was managed in our primary care clinic. He presented several times with refractory hypertension (SBP $>140 /$ DBP $>100$ ) which could not be controlled with four antihypertensive medications including Lisinopril, hydrochlorothiazide, amlodipine and atenolol. We started looking for secondary causes of HTN based on his refractory blood pressure on 4-antihypertensive medications including diuretic. He underwent renal ultrasound investigation which demonstrated normal sized kidneys with no renal disease.

The patient's aldosterone to renin ratio was calculated on January 2020 by obtaining plasma renin activity and aldosterone from the blood of the patient taken in midmorning from the patient in seated position and it was 19.2 with aldosterone level of $14.6 \mathrm{ng} / \mathrm{dl}$ and plasma renin activity of $0.759 \mathrm{ng} / \mathrm{ml} / \mathrm{hr}$ decreased despite the use of ACE-inhibitors. This made us think about Primary hyperaldosteronism as a cause of his resistant HTN. We changed at that time the Lisinopril to Hydralazine.

The patient was found also to have unequal SBP readings in both arms-right $160 \mathrm{~mm} \mathrm{Hg}$ and left $145 \mathrm{~mm} \mathrm{Hg}$ at a follow up visit and therefore underwent CT angiogram/CTA/ of the chest, abdomen, and pelvis. CTA of the chest was only notable for an incidental right subscapular lipoma. The CT / CTA of the abdomen and pelvis was read as negative with no evidence of renal artery stenosis, however there was sub centimeter focus on right adrenal gland suspicious for adenoma with 8 HU on non-contrast CT and contrast washout more than $60 \%$ in 15 minutes all suggestive of lipid rich adenoma of the right adrenal gland. We checked also free metanephrines and nor metanephrines in the blood and they were normal as well as we performed $1 \mathrm{mg}$ overnight Dexamethasone suppression test with measuring 9 a.m. cortisol - $1.2 \mathrm{mcg} / \mathrm{dl}-$ normal suppression.

A repeat aldosterone to renin ratio was obtained in June, 2020 and was significantly elevated (compared to the levels obtained 5 months prior) at 121-elevated with an aldosterone level of $21.9 \mathrm{ng} / \mathrm{mL}$-elevated and plasma renin 


\section{Open Access Journal of Endocrinology}

activity of $0.181 \mathrm{ng} / \mathrm{mL} / \mathrm{hr}$-suppressed. We believe that the first reading of aldosterone to plasma renin activity ratio which was not significantly elevated and not significantly increased aldosterone were due to the use of ACE inhibitors used prior to the test in January, 2020. The patient had lownormal Potassium level in the blood-3.5-3.7mek/l.

The patient underwent confirmatory test for primary hyperaldosteronism with oral sodium load with $200 \mathrm{mmol}$ sodium diet per day for 3 days and the measured aldosterone in 24 hour urine was still high at $20 \mathrm{mcg} /$ day with sodium in the urine of $250 \mathrm{mmol} /$ day. His Potassium level in the blood was normal, he did not have cardiac insufficiency or arrhythmia and BP was 150-155/95-100 during the test. This confirmed primary hyperaldosteronism diagnosis.
To find out what is the cause of primary hyperaldosteronism we performed an MRI of the abdomen with and without contrast was ordered for further evaluation given these findings and confirmed a $7 \mathrm{~mm}$ right adrenal nodule consistent with an adrenal adenoma. Then the patient underwent bilateral adrenal vein sampling, with Cosyntropin stimulation and findings were consistent with primary hyperaldosteronism (Tables 1 \& 2). The selectivity index which is the ratio of cortisol in the adrenal vein blood to cortisol in the systemic venous blood from vena cava inferior was above 3 above $630 \mathrm{mcg} / \mathrm{dl}$ in both left and right adrenal veins with systemic cortisol level of $18.8-23.1 \mathrm{mcg} /$ dl. This showed that the catheterization of both adrenal veins was adequate and the usually difficult right adrenal vein was successfully cannulated.

\begin{tabular}{|c|c|c|c|}
\hline Sample Number & Systemic Cortisol Level & Left Adrenal Vein Cortisol Level & Right Adrenal Vein Cortisol Level \\
\hline 1 & 18.8 & $>630$ & $>630$ \\
\hline 2 & 19.3 & $>630$ & $>630$ \\
\hline 3 & 23.1 & $>630$ & $>630$ \\
\hline 4 & 22.6 & $>630$ & $>630$ \\
\hline
\end{tabular}

Table 1: Systemic Cortisol with normal reference range of $6.2-19.4 \mathrm{mcg} / \mathrm{dl}$.

\begin{tabular}{|c|c|c|}
\hline Sample Number & Left Adrenal Vein Aldosterone Level & Right Adrenal Vein Aldosterone Level \\
\hline 1 & 1575 & 8982 \\
\hline 2 & 1651.2 & 8518.3 \\
\hline 3 & 1417.7 & 8764 \\
\hline 4 & 1270.3 & 7221 \\
\hline
\end{tabular}

Table 2: Aldosterone Units of measurement is $\mathrm{ng} / \mathrm{dL}$.

The lateralization index which was calculated by dividing the aldosterone to cortisol ratio in the dominant right adrenal vein by that in the non-dominant left adrenal vein in our case was above 4 -5-6 in our patient. This confirmed that the adenoma in the right adrenal gland was producing excessive amounts of aldosterone. Now that a definitive cause of hypertension had been made, a potassium-sparing diuretic was added to the medication regimen and the Hydralazine dose was decreased with improvement of patient's blood pressure. We referred the patient to endocrine surgeon for removal of the right adrenal ald osterone producing adenoma.

\section{Discussion}

The main function of aldosterone is to increase the number of open sodium and potassium channels in the principal cells of the cortical collecting tubule to increase sodium reabsorption leading to water retention and hypertension and potassium loss leading to hypokalemia. The classical presenting signs of primary hyperaldosteronism are hypertension and hypokalemia, but the potassium levels are frequently normal. With this the plasma potassium concentration stabilizes at a lower level and hypokalemia does not occur unless an external factor like a diuretic therapy is added $[2,6,7]$.

The diagnosis of primary hyperaldosteronism starts with the clinical suspicion and measuring the morning plasma renin activity and Aldosterone levels in sitting position in mid-morning and then estimating aldosterone to plasma renin activity ratio in the blood and aldosterone level in the blood. If the ratio is elevated-usually above 20 with increased Aldosterone level above $15 \mathrm{ng} / \mathrm{dl}$ then we perform in most instances confirmatory test with suppression protocols with IV saline or oral sodium load in USA. Those tests should not be performed in severe uncontrolled blood pressure, hypokalemia, cardiac insufficiency or arrhythmia.

There are a lot of medications which lead to either falsely positive ratio of aldosterone to plasma renin activity 
like alfa-2 or beta-blockers, direct renin inhibitors or NSAID's or falsely negative ratio like ACE/ARB inhibitors, diuretics, SSRI's, mineralocorticoid receptor antagonists (MRA). MRA should be stopped before the aldosterone to plasma renin activity ratio is measured for 4-6 weeks, but the other medications can be continued if the ratio is above 20 and plasma renin activity is suppressed and aldosterone is increased like in our case.

After confirming primary hyperaldosteronism, the next step is to find the cause by performing adrenal CT or MRI to figure out if the cause is bilateral adrenal hyperplasia or adrenal producing tumor most likely adenoma. However, the absence of a mass does not exclude an aldosterone producing tumor. Aldosterone producing adenomas (APAs) can be very small [eg, $<3 \mathrm{~mm}$ in diameter] and lesions less than $1 \mathrm{~cm}$ in diameter may be missed on CT. Usually aldosterone producing adenomas are small-less than 2-2.5 sm. Bilateral lesions of the adrenal glands are not diagnostic of hyperplasia (because some patients with an aldosteronoma in one adrenal gland have a nonfunctioning adrenal nodule in the other] usually in patient above the age of 35 and the patient might have unilateral adrenal hyperplasia with hyperaldosteronism from one gland and inactive adenoma in the other adrenal gland.

This is why we perform adrenal vein sampling with Cosyntropin stimulation in most instances as described above and if there is lateralization of the aldosterone production to one of the adrenal glands the cause of primary hyperaldosteronism is aldosterone producing tumor or unilateral hyperplasia. If the ratio aldosterone/cortisol in both glands is similarly increased and if it is usually 2-3 times higher than in the vena cava inferior/systemic circulation/ the cause of primary hyperaldosteronism is bilateral adrenal hyperplasia $[1,8,9]$.

Treatment of primary hyperaldosteronism caused by adrenal aldosterone producing tumor or unilateral aldosterone producing hyperplasia is usually laparoscopic unilateral adrenalectomy. This usually results in normalization of the hypokalemia and improvement in hypertension in 30-60\% of patients if diagnosis is made early. In primary hyperaldosteronism, due to Bilateral adrenal hyperplasia or patients with unilateral adrenal producing tumor who refuses surgical management the treatment is with mineralocorticoid receptor antagonists.

\section{References}

1. Funder JW, Carey RM, Mantero F, Murad MH, Reincke M, et al. (2016) The management of primary aldosteronism: case detection, diagnosis and treatment: an endocrine society practice guideline. J Clin Endocrinology and Metab 101(5): 1889-1916.

2. Young WF (2007) Primary aldosteronism: renaissance of a syndrome. Clinical Endocrinology (Oxf) 66(5): 607618.

3. Rossi GP, Sacchetto A, Visentin P, Canali C, Graniero GR, et al. (1996) Changes in left ventricular anatomy and function in hypertension and primary aldosteronism. Hypertension 27(5): 1039-1045.

4. Savard S, Amar L, Plouin F, Steichene O (2013) Cardiovascular complications associated with primary aldosteronism: a controlled cross-sectional study. Hypertension 62(2): 331-336.

5. Millez P, Girerd X, Plouin PF, Blacher J, Safar ME, et al. (2005) Evidence for increased rate of cardiovascular events in patients with primary aldosteronism. J Am Coll Cardiol 45(8): 1243-1248.

6. Brilla CG, Weber KT (1992) Mineralocorticoid excess, dietary sodium, and myocardial fibrosis. J Lab Clin Med 120(6): 893-901.

7. Gyamlani G, Headley CM, Naseer A, Valaulikar GS, Geraci SA (2016) Primary aldosteronism: diagnosis and management. Am J Sci 352(4): 391-398.

8. Rossi GP, Auchus RJ, Brown M, Lenders JWM, Naruse $M$, et al. (2014) An expert consensus statement on the use of adrenal vein sampling for subtyping of primary aldosteronism. Hypertension 63(1): 151-160.

9. Dakkers T, Deinum J, Schultzekool LJ, Blondin D, Vonend 0 , et al. (2013) Plasma metanephrine for assessing the selectivity of adrenal vein sampling. Hypertension 62(6): 1152-1157. 